\title{
Modelo mecánico para el cálculo de la resistencia a cortante de forjados de vigueta y bovedilla
}

\author{
Mechanical model for calculating the shear strength \\ of beam-and-block floors
}

$\underline{\text { C. Ribas }}^{(*)}$, A. Cladera $\left.{ }^{*}\right)$

RESUMEN

El presente estudio plantea la evolución de un modelo de cálculo de resistencia a cortante existente en la bibliografía, para su aplicación a forjados de vigueta pretensada y bovedilla. El modelo previo evalúa la resistencia a cortante a partir de un área efectiva a cortante sobre una posición determinada de la fibra neutra. El nuevo modelo presenta como mejora principal, además de la interacción momento-cortante, la evaluación del efecto que tiene la capa de compresión en la resistencia a cortante, hecho que no se tiene en cuenta en la Instrucción EHE-o8. Las predicciones del modelo se comparan con los resultados obtenidos en dos campañas experimentales de forjados de vigueta y bovedilla, obteniendo una correlación muy satisfactoria. Además, se observa que el modelo generaliza correctamente los resultados para otras tipologías de vigas, al compararlo con los resultados de campañas experimentales de vigas en T obtenidos de la bibliografía científico-técnica.

Palabras clave: Forjados de vigueta y bovedilla; vigas en T; hormigón, resistencia a cortante.

\section{ABSTRACT}

This paper presents the evolution of a model for calculating shear strength that already exists in the literature, for its application to beam-and-block floors. The previous model evaluates the shear strength based on a shear effective area deduced from a determined position of the neutral axis. The new model presents as major improvement, in addition to bending-shear interaction, the evaluation of the effect that the compressed flanges of a T-beam have in the shear strength, a fact that is not taken into account in the designing method of the EHE-o8 Code. Model predictions are compared with the results of two experimental campaigns on beam-and-block floors, resulting a very satisfactory correlation. It is further noted that the model fits well with the experimental results of T-beams obtained from the technical literature.

Keywords: Beam-and-blockfloors; T-beams; concrete; shear strength.

(*) Universitat de les Illes Balears, Palma de Mallorca (España).

Persona de contacto/Corresponding author: carlos.ribas@uib.es (C. Ribas)

Cómo citar este artículo/Citation: Ribas, C., Cladera, C. (2014). Modelo mecánico para el cálculo de la resistencia a cortante de forjados de vigueta y bovedilla. Informes de la Construcción, 66(EXTRA-1): mo10, doi: http://dx.doi.org/10.3989/ic.13.114.

Licencia / License: Salvo indicación contraria, todos los contenidos de la edición electrónica de Informes de la Construcción se distribuyen bajo una licencia de uso y distribución Creative Commons Reconocimiento no Comercial 3.o. España (cc-by-nc). 


\section{INTRODUCCIÓN}

El hormigón pretensado y prefabricado está ampliamente extendido alrededor del mundo, y el hecho de que los forjados de vigueta pretensada y bovedilla fueron una de las primeras aplicaciones del hormigón pretensado, no implica que hayan caído en desuso (1) (2). Lejos de ello, los forjados de vigueta pretensada y bovedilla se siguen utilizando alrededor del mundo. Si bien la resistencia a flexión de los forjados de vigueta pretensada y bovedilla se encuentra perfectamente caracterizada, este tipo de forjados tiene una serie de características que lo convierten en un elemento complejo a la hora de obtener su resistencia a cortante:

- La existencia de dos hormigones en la sección, que además coexisten en una misma fibra horizontal.

- La sección está construida evolutivamente.

- La sección tiene una parte pretensada prefabricada, y otra in situ sin pretensar.

- La geometría de la sección no sólo tiene forma de T, sino que además el ancho del alma y su proporción entre hormigón pretensado y hormigón in situ varían a lo largo del alma.

- Las proporciones de su sección son muy achatadas: $b / h \approx$ 2,5 ; donde $b$ es el ancho máximo de la sección y $h$ es el canto del forjado.

Por otra parte, la resistencia a cortante de las vigas de hormigón armado con sección en forma de $\mathrm{T}$ y sin armadura transversal es evaluada en la mayor parte de normativas de forma simplificada, igualándola a la resistencia a cortante que tendría una viga rectangular de ancho igual al del alma de la sección, enfoque similar al planteado por la Instrucción EHE-08 (3) tanto para vigas con sección en T como para forjados de vigueta pretensada y bovedilla. Sin embargo, desde los años 50 muchos modelos tanto empíricos como racionales evalúan el incremento de resistencia a cortante que supone la existencia de alas comprimidas en la sección en forma de T (4) (5) (6). Entre los citados modelos cabe destacar el presentado por Zararis et al. en (6) y (7), modelo en el que se basa el modelo presentado en este artículo. El modelo propuesto se puede clasificar dentro del grupo de modelos que se basan en que el mecanismo fundamental resistente a cortante es el de las tensiones tangenciales que soporta el bloque de hormigón comprimido a flexión que está por encima de la fibra neutra. Estos modelos adquieren especial interés en las vigas con sección en T, y por tanto para los forjados de vigueta pretensada y bovedilla, pues tienen una zona comprimida a flexión más amplia que la de una sección rectangular. Además, en las vigas con sección en $\mathrm{T}$ la altura de la fisuración a flexión es mayor que en las vigas de sección rectangular, y por tanto, los anchos de fisura son mayores y el mecanismo resistente cortante-fricción se ve debilitado. Kotsovos et al. (8) realizaron unos ensayos a cortante en vigas con sección en $\mathrm{T}$ en los que se mostraba que el ancho de fisura era muy elevado para que se pudiesen desarrollar los mecanismos resistentes a través de la fisura: cortante-fricción y tracciones residuales.

Este enfoque de que la mayor parte del cortante se transmite en la zona comprimida y que en la zona fisurada la transmisión de cortante es prácticamente nula ya se ha planteado para otro tipo de elementos (9) (10). En cualquier caso, también es cierto que en modelos más refinados, que requieren de un coste computacional elevado, no se acostumbra plantear que toda la resistencia a cortante se deba a las tensiones en la cabeza comprimida, sino que se tienen en cuenta todas las distintas contribuciones a la resistencia a cortante (11).

En 2013, con el fin de evaluar la resistencia a cortante de los forjados de vigueta pretensada y bovedilla se publicaron los resultados de dos campañas de ensayos en forjados de vigueta pretensada y bovedilla (1) y (2), de 3 elementos realizada en el 2007 y de 24 elementos realizada en el 2010 (Figura 1), estas campañas se presentan brevemente en el punto 4. En este estudio se utilizarán los datos experimentales obtenidos en las citadas campañas para evaluar la bondad del método propuesto.

\section{MODELO DE ZARARIS ET AL.}

A continuación se resume el modelo de comportamiento de las vigas de hormigón armado sometidas a esfuerzo cortante, propuesto por Zararis et al. (6) (7), al ser este modelo la base para el modelo propuesto en el presente artículo. No se presenta la parte del modelo para elementos con armadura a cortante al no ser de interés para el presente estudio.

\subsection{Fisura crítica}

En base a la observación de las roturas a cortante de la bibliografía técnica consultada, Zararis et al. (6) y (7) dividen la fisura crítica a cortante en dos ramas principales (Figura 2a). La primera aparece inicialmente debido a las tracciones inducidas por la flexión del elemento, tiene una pendiente superior a $45^{\circ}$ con respecto a la horizontal y su altura viene delimitada por una línea que va desde el apoyo más cercano a la carga al centro de gravedad de las compresiones producidas por flexión en el hormigón $\mathrm{C}_{\mathrm{f}}$ (Figura 2a), en la sección de aplicación de la carga. En esta primera rama se hace imposible el desarrollo del mecanismo de engranamiento de áridos o cortante-fricción dado el elevado ancho entre fisuras (1) (2). La segunda rama se extiende desde el extremo de la rama anterior hasta $\mathrm{C}_{\mathrm{f}}$. El trazado de las dos ramas se puede ver de forma esquemática en la Figura $2 \mathrm{a}$.

\subsection{Mecanismo resistente}

Zararis y Papadakis (7) presentan una posible disposición de las fuerzas que actúan en la región de la viga donde se produce la división de hormigón que se muestra en la Figura 2b. La resultante de las fuerzas $\mathrm{P} \mathrm{y}_{\mathrm{f}}$ se pueden descomponer en $\mathrm{F}_{1}$ $\mathrm{y} \mathrm{F}_{2}$, cada una de los cuales representa la resultante de tensiones que actúan por encima y por debajo de la segunda rama de la fisura crítica, respectivamente. La fuerza F1 equilibra

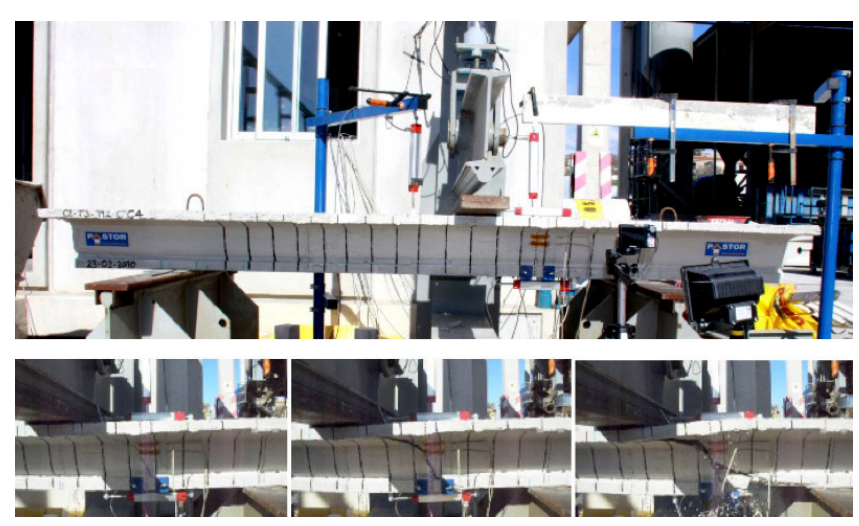

Figura 1. Configuración de ensayo y rotura a cortante (1). 


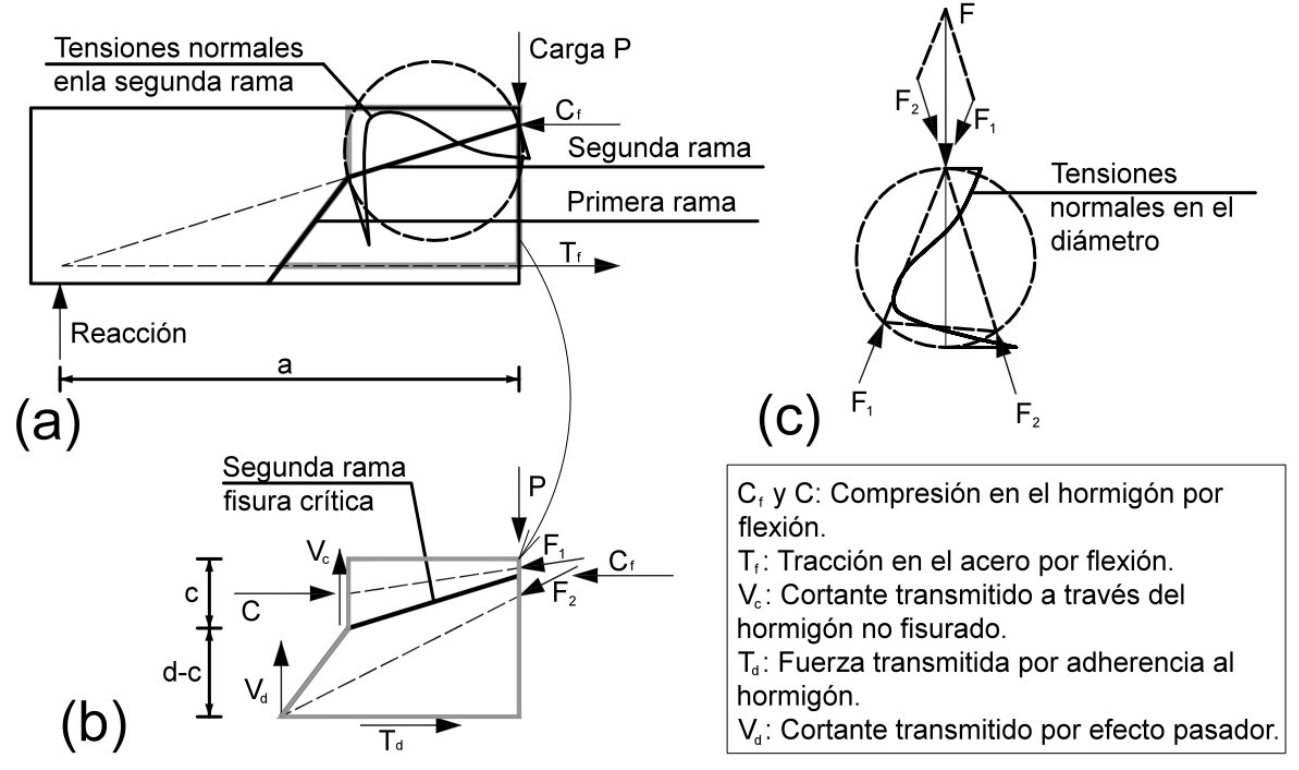

Figura 2. (a)Trazado de las ramas y distribución de las tensiones normales a lo largo de la segunda rama de la fisura crítica; (b)Propuesta de equilibrio de fuerzas; (c)Esquema del cilindro apoyado en dos puntos y tensiones normales en el diámetro. Adaptada de (7).

la resultante de las fuerzas $\mathrm{C} \mathrm{y} \mathrm{V}_{\mathrm{c}}$ en la zona de compresión, mientras que la fuerza $\mathrm{F} 2$ equilibra la resultante de las fuerzas $\mathrm{T}_{\mathrm{d}}$ (fuerza transmitida por adherencia de la armadura longitudinal al hormigón) y $\mathrm{V}_{\mathrm{d}}$ (fuerza transmitida por el efecto pasador al hormigón). Por lo tanto, como una primera aproximación, la región de la viga, donde se produce la segunda rama de la fisura crítica con las fuerzas correspondientes (Figura $2 b$ ), puede ser representada por un disco circular apoyado en dos puntos en la parte inferior, como se muestra en la Figura 2c, en donde las tensiones normales en la línea de rotura guardan cierto parecido con las que se producen en un ensayo de tracción indirecta. Debido a esta similitud, Zararis y Papadakis (7) deducen que la resistencia a cortante de una viga de hormigón sin armadura a cortante, es igual al área de hormigón que queda por encima de la primera rama de la fisura crítica, es decir por encima de la fibra neutra, multiplicada por la resistencia a tracción indirecta del hormigón. Por tanto en una viga rectangular, la resistencia a cortante sería:

$$
V_{c r}=b \cdot c \cdot f_{c t}=A_{e f, v} \cdot f_{c t}
$$

donde $b$ sería el ancho de la sección, $c$ la profundidad del bloque comprimido a flexión, $f_{c t}$ la resistencia a tracción indirecta del hormigón y $A_{e f, v}$ el área eficaz a cortante. Para $f_{c t}$ Zararis et al. adoptan el valor de $f_{c t}=0,3 f_{c k}{ }^{2 / 3}$. Obsérvese que Zararis et al. obvian el posible efecto de cortante fricción que se produce en la primera rama de la fisura crítica.
Para las secciones en T, Zararis et al. (6) proponen una simplificación para hallar $c$, a partir de las ecuaciones de equilibrio de fuerzas de la sección, sometiendo a la fibra superior a una deformación de compresión del hormigón $\varepsilon_{c o}=0,002$, y adoptando un diagrama tensión-deformación rectangular del hormigón, como se muestra en la Figura 3a.

\subsection{Efecto tamaño y resistencia de vigas con sección en $T$}

Zararis y Papadakis (7) proponen añadir a la ecuación [1], un factor para evaluar el efecto tamaño, quedando la expresión:

$$
V_{c r}=\xi A_{e f, v} \cdot f_{c t}
$$

Donde el efecto tamaño se determina a partir de la longitud del vano a cortante $a$, que es la distancia del apoyo a la sección de aplicación de la carga, y el canto útil de la sección de la viga $d$ :

$$
\xi=1,2-0,2 \frac{a}{d} d \geq 0,65
$$

Basándose en la observación de la fisuración diagonal en las alas de las vigas ensayadas, Zararis et al. proponen que el área efectiva a tener en cuenta de las alas de una sección en T, para el cálculo de la resistencia a cortante, tenga la forma de embudo mostrada en la Figura 3 b.

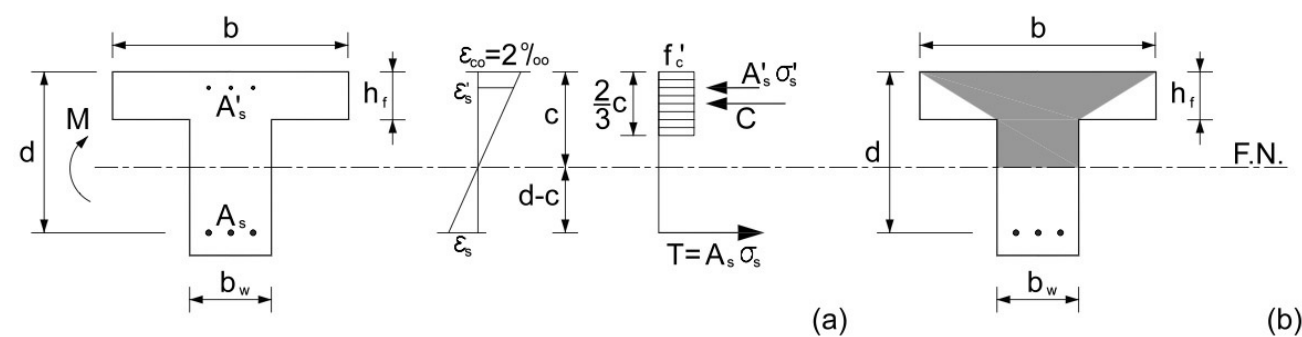

Figura 3. (a) Distribución de deformaciones y tensiones en una sección en T; (b) área efectiva a cortante del hormigón, sombreada. Adaptada de (6). 


\section{ENFOQUE NORMATIVO}

La Instrucción EHE-o8 (3) distingue para el cálculo del esfuerzo de agotamiento a cortante, si el elemento ha fisurado, o no, a flexión. Si el elemento no ha fisurado a flexión, el esfuerzo de agotamiento a cortante se obtiene de una formula deducida a partir del círculo de Mohr.

Si el elemento ha fisurado a flexión, situación habitual en el cálculo cuotidiano de forjados de vigueta y bovedilla, la Instrucción EHE-o8 (3) trata de forma particular los forjados unidireccionales de vigueta pretensada, aplicando de forma singular la formulación general para el esfuerzo de agotamiento a cortante, dada por la ecuación [4], y el valor mínimo de la resistencia a cortante dada por la ecuación [5].

$$
\begin{gathered}
V_{u 2}=\left[\frac{0,18}{\gamma_{c}} \xi\left(100 \rho_{l} f_{c v}\right)^{1 / 3}-0,15 \alpha_{1} \sigma_{c d}\right] b_{0} d \\
V_{u 2}=\left[\frac{0,075}{\gamma_{c}} \xi^{3 / 2} f_{c v}^{1 / 2}-0,15 \alpha_{1} \sigma_{c d}\right] b_{0} d
\end{gathered}
$$

Donde: $f_{c v}$ es la resistencia efectiva del hormigón a cortante cuyo valor puede tomarse como la resistencia a compresión del hormigón $f_{c k}$ en MPa y no mayor de $60 \mathrm{MPa} ; \xi=1+(200 / \mathrm{d})^{1 / 2}$ ; $d$ es el canto útil en mm de la sección referido a la armadura longitudinal de flexión siempre que ésta sea capaz de resistir el incremento de tracción producido por la interacción cortante-flexión; $b_{o}$ es el ancho del alma si se trata de un elemento con sección en T; $\sigma_{c d}$ es la tensión axial media en el alma de la sección (compresión positiva); $\rho_{l}=\left(A_{S}+A_{p}\right) /$ $\left(b_{o} d\right)$ es la cuantía geométrica de la armadura longitudinal principal de tracción, pasiva y activa adherente, anclada a una distancia igual o mayor que $d$ a partir de la sección de estudio; y $\alpha_{1}$ es un factor que tiene en cuenta la introducción progresiva de las tensiones transmitidas por el pretensado al hormigón en el caso de piezas pretensadas con anclaje por adherencia (3).

En los forjados con vigueta pretensada prefabricada, el cortante de agotamiento por tracción en el alma según la Instrucción EHE-08 será el menor de los valores obtenidos considerando por una parte el ancho mínimo del nervio pretensado y por otra el menor ancho del hormigón vertido en obra. Por lo tanto la Instrucción EHE-o8 iguala el comportamiento de los forjados al de la sección menos resistente de las dos secciones rectangulares sombreadas en las Figura 4. Resulta evidente que este planteamiento contrasta con el enfoque planteado por Zararis et al.

En el primer caso, ver Figura 4a, se considerará como valor de cálculo de la resistencia efectiva a cortante del hormigón $f_{c v}$ el correspondiente a la vigueta pretensada, como tensión $\sigma_{c d}$ la referida al área de la vigueta, y como cuantía geométrica de armadura $\rho_{l}$ la referida a una sección rectangular de ancho $b_{o}=b_{1}$ mostrado en la Figura 4a, es decir el ancho mínimo del nervio, y al canto útil $d$.

En el segundo caso, ver Figura 4b, se considerará como resistencia efectiva a cortante del hormigón $f_{c v}$ la del hormigón
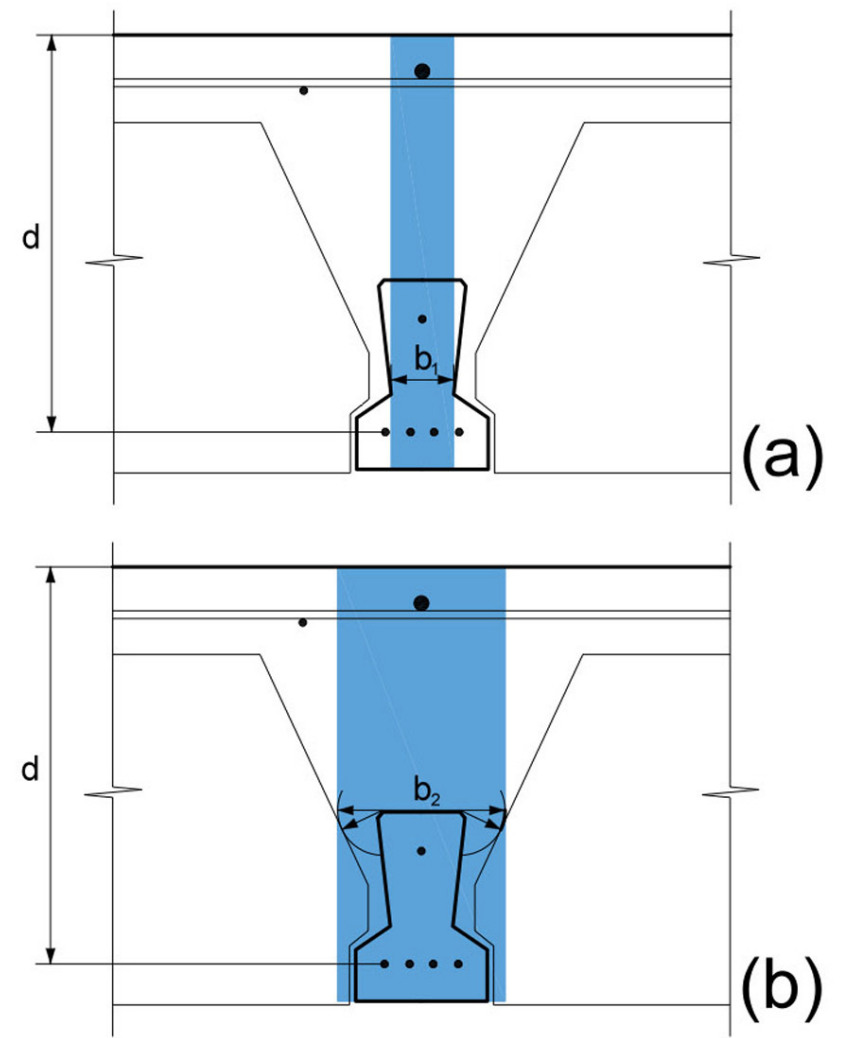

Figura 4. Secciones de las dos comprobaciones según la Instrucción EHE-o8, adaptada de (3).

vertido in situ, se considerará nula la tensión $\sigma_{c d}$, y la cuantía geométrica de armadura $\rho_{l}$ se referirá a una sección rectangular de ancho $b_{o}$ y canto $d$, siendo $b_{o}=b_{2}$ mostrado en la Figura $4 \mathrm{~b}$, es decir la distancia mínima recorrida entre bovedillas a través de la zona del hormigón vertido in situ (3).

\section{DATOS EXPERIMENTALES}

\subsection{Campañas de forjados de vigueta pretensada y bovedilla}

Las variables estudiadas en las campañas experimentales (1) (2) fueron: el canto del nervio del forjado (20, 24, 25, 29 y $30 \mathrm{~cm}$ ), el área de pretensado en la vigueta prefabricada (T3: $79,48 \mathrm{~mm}^{2}$ y T5: $117,72 \mathrm{~mm}^{2}$ ), la geometría de la vigueta pretensada (vigueta autoportante T20 de $20 \mathrm{~cm}$ de canto y semivigueta T12 de $12 \mathrm{~cm}$ de canto), la existencia de la capa de compresión así como la influencia de su espesor (sin capa de compresión SCC, con capas de compresión de 4, 5, 7 y $8 \mathrm{~cm}$, $\mathrm{CC}_{4}, \mathrm{CC}_{5}, \mathrm{CC}_{7}$ y $\mathrm{CC} 8$ respectivamente).

La nomenclatura de los especímenes en ambas campañas se plantea según las variables (separadas por guiones) estudiadas en la segunda campaña, tal y como se muestra en la Tabla 1 y la Figura 5.

Del análisis de las campañas de forjados de vigueta pretensada y bovedilla cabe destacar dos conclusiones fundamentales para el desarrollo del modelo que se propondrá más adelante:

Tabla 1. Nomenclatura de los especímenes, atendiendo a sus variables.

\begin{tabular}{|c|c|c|c|}
\hline Canto total (cm) & Tipo de armado & Tipo de vigueta pretensada (cm) & Espesor capa de compresión (cm) \\
\hline $20-24-25-29-30$ & $\mathrm{~T}_{3}-\mathrm{T}_{5}$ & $\mathrm{~T} 12-\mathrm{T} 2 \mathrm{O}$ & $\mathrm{SCC}-\mathrm{CC}_{4}-\mathrm{CC} 7$ \\
\hline
\end{tabular}




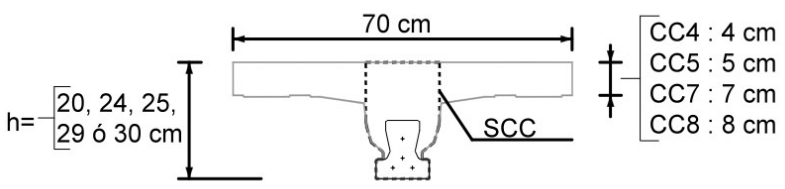

(a)

(b)
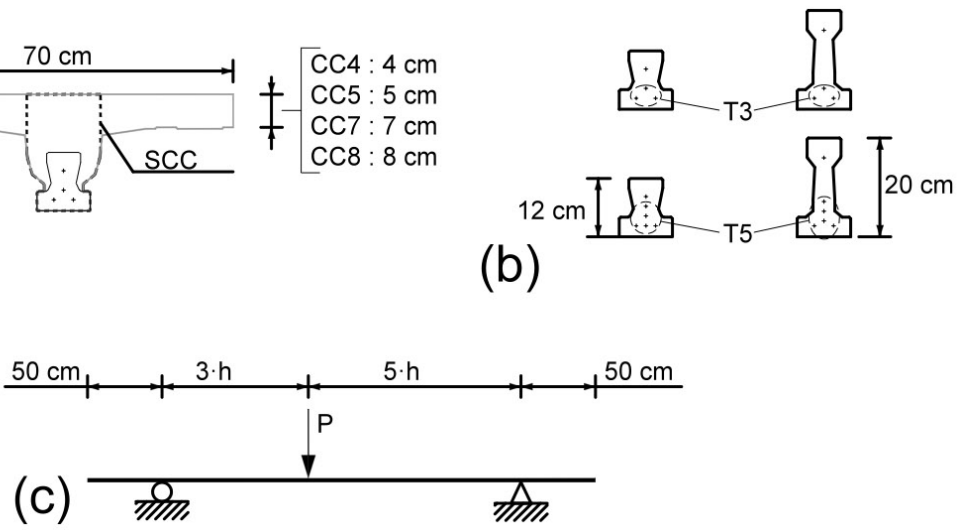

Figura 5. Configuración de las características más significativas de las campañas experimentales (1) (2) (a)Diferentes cantos y capas de compresión ensayadas; (b)Viguetas T20 y T12 (20 y $12 \mathrm{~cm}$ de canto respectivamente); (c) Disposición de la carga, donde h es el canto de la viga.

- La forma de la fisura crítica a cortante se puede separar en dos ramas claramente diferenciadas cuya geometría coincide con la descrita por Zararis et al. (6) (7) y ha sido descrita en el apartado 2.1. El colapso de la viga se produce cuando la segunda rama llega a la sección de aplicación de la carga (1).

- El ancho de fisura en la primera rama de la fisura crítica a cortante, en un estado previo a la rotura, es mucho mayor que el necesario para desarrollar el engranamiento de áridos o cortante-fricción (1) (2) según las teorías desarrolladas por Fenwick y Paulay (12) o por Walraven (13).

\subsection{Datos experimentales de campañas en vigas con sección en $T$ obtenidos de la bibliografía}

Dado que en la bibliografía no se encuentran ensayos de forjados de vigueta y bovedilla pretensada, se ha seleccionado de la bibliografía campañas de ensayos de vigas de hormigón armado con sección en T y sin armadura a cortante. Estos ensayos permitirán comprobar la capacidad de generalización del modelo a otras secciones más genéricas que las inicialmente planteadas. Las fuentes consultadas son:

- Ferguson y Thompson 1953 (14), 24 vigas.

- Al-Alusi 1957 (4), 25 vigas.

- Placas y Regan 1971 (15), 7 vigas.

- Kani 1979 (16), 178 vigas, sólo las vigas con sección en T.

- Taylor et al. 1980 (17), 1 viga.

- Palaskas et al. 1981 (18), 4 vigas.

Todos estos ensayos también figuran en la base de datos presentada por Collins et al. en 2008 (19). Un total de 239 vigas han sido revisadas de las campañas arriba citadas, de las cuales se han seleccionado para el análisis 188. Los parámetros a satisfacer para la selección han sido:

- Las vigas que tengan sección en $\mathrm{T}$.

- Las vigas en las que $a / d \geq 2,5$ con el fin de evitar las que tengan efecto arco, donde $a$ es la distancia desde el apoyo al punto de aplicación de la carga.

- Las vigas que han roto a cortante.

- Las vigas ensayadas con las alas de la sección en T comprimidas por flexión. Por este motivo se han eliminado 3 vigas de la campaña de Al-Alusi en 1957 que se ensayaron invirtiendo la viga en $\mathrm{T}$.

\section{MODELO PROPUESTO}

En el presente estudio, gracias al planteamiento de equilibrio en la sección mediante un análisis de fibras, se obtienen las tensiones normales en la sección. Repitiendo el proceso para diferentes posiciones de la fibra neutra se consigue evaluar la relación momento-curvatura. El modelo propuesto se centra en una sección de análisis determinada. La sección de análisis planteada está situada a $0,5 d$ del punto de aplicación de la carga, pues se sitúa en la parte central de la segunda rama de la fisura crítica observada por Zararis y Papadakis (7). El análisis planteado se puede realizar a ambos lados del punto de aplicación de la carga, en el caso de ensayos no simétricos, puesto que la interacción de los esfuerzos solicitados y el factor tamaño de Zararis y Papadakis dependen de la longitud del vano a cortante $a$. Cabe destacar que las campañas experimentales sobre forjados de viguetas y bovedillas que se han descrito en el apartado anterior se llevaron a cabo con la carga desplazada (Figura 5c), por lo que el análisis planteado se puede realizar, en este caso, tanto por el lado largo como por el lado corto de las vigas ensayadas.

A partir del análisis momento-curvatura se obtiene la posición de la fibra neutra para cada momento flector solicitante, y gracias a ello se deduce el área efectiva a cortante $A_{e f, v}$, en base al área resistente definida en la Figura 3b. Este área efectiva multiplicada por la resistencia a tracción indirecta $f_{c t}$ y por el factor que tiene en cuenta el efecto tamaño ecuación [3], es la resistencia a cortante planteada. Todo junto permite plantear un cortante último para cada momento flector concomitante y generar así un diagrama de interacción momento-cortante. Para el valor de $f_{c t}$ se ha tomado el sugerido por Collins y Mitchell en (20) $f_{c t}=0,33 \cdot f_{c k}^{1 / 2}$.

Un ejemplo del análisis planteado para el elemento 29-T5T12-CC4 se presenta en la Figura 6, en ella se observa que:

- Para momentos menores al de fisuración, el área efectiva a cortante es constante, y por tanto la resistencia a cortante del elemento también es constante, línea continua azul horizontal en la Figura 6.

- Una vez iniciada la fisuración, a cada momento flector le corresponde una fibra neutra, y por tanto una resistencia a cortante diferente, línea continua azul no horizontal.

- La interacción momento cortante no puede superar el momento último, línea azul discontinua. 
- La predicción del cortante último según el modelo previo de Zararis et al., no depende del momento flector aplicado, línea verde de 3 rayas y 2 puntos. En el modelo propuesto, sí que se considera la interacción entre ambos esfuerzos.

- No se puede solicitar al elemento más allá de la línea vertical continua negra, que representa el momento flector que solicita a la sección de análisis, en el instante de la rotura por flexión de la sección bajo la aplicación de la carga, que es la sección más solicitada a flexión.

- La solicitación de la viga en la sección de análisis se presenta mediante línea discontinua roja inclinada. Si esta línea corta antes a la línea continua azul, significa que rompe por cortante (rombo azul), mientras que si corta antes a la línea vertical negra quiere decir que el elemento rompe por flexión (que no es el caso representado en la Figura 6).

- El par de esfuerzos de rotura observados en el ensayo están representados con un cuadrado rojo.

\section{COMPARACIÓN CON LOS RESULTADOS EXPERIMENTALES}

\subsection{Comparación del modelo propuesto con los resultados experimentales en forjados de vigueta pretensada y bovedilla}

En la Tabla 2 se muestra:

- Las cargas de rotura y el tipo de rotura observado en los ensayos ( $S$ : Rotura cortante lado corto, $F$ : rotura a flexión y $S L$ : rotura a cortante lado largo).

- En el segundo bloque, referido a las predicciones de la Instrucción EHE-08, se presentan las cargas últimas predichas por la Instrucción de rotura a flexión y cortante por el lado corto, y la más desfavorable de estas dos. A continuación se muestra el cociente entre la carga última experimental y la carga última predicha por la Instrucción EHE-o8.

- En el tercer bloque se presentan los valores predichos por el modelo propuesto en este artículo, es decir, las cargas últimas de rotura a flexión, cortante por el lado corto y cortante por el lado largo, y la más desfavorable de estas tres. A continuación se muestra también el cociente entre la carga última experimental y la carga última predicha por la propuesta.

La Instrucción EHE-o8 no plantea que la resistencia a cortante varíe en función del momento flector concomitante, por lo que en la Tabla 2 sólo se presenta la carga última a cortante por el lado corto que es el más solicitado.

La Tabla 2 muestra que según la propuesta las cargas de rotura por cortante y flexión están más cercanas entre sí que las previstas por la Instrucción EHE-o8.

Las predicciones de carga última a cortante previstas por la EHE-o8 dentro de las series A a $\mathrm{H}$ son iguales, puesto que la Instrucción obvia el efecto beneficioso del espesor de la capa de compresión. Sin embrago, la propuesta sí que prevé una resistencia creciente dentro de cada una de las citadas series a medida que aumenta el espesor de la capa de compresión.

La Tabla 2 presenta los cocientes entre las cargas de rotura previstas por la Instrucción EHE-08 y la carga de rotura experimental, el promedio de estos cocientes es de 1,78, la desviación estándar de 0,41 y el coeficiente de variación del 23,2 \%. Una desviación tan alta y un promedio tan por el lado de la seguridad están producidos por un exceso de seguridad

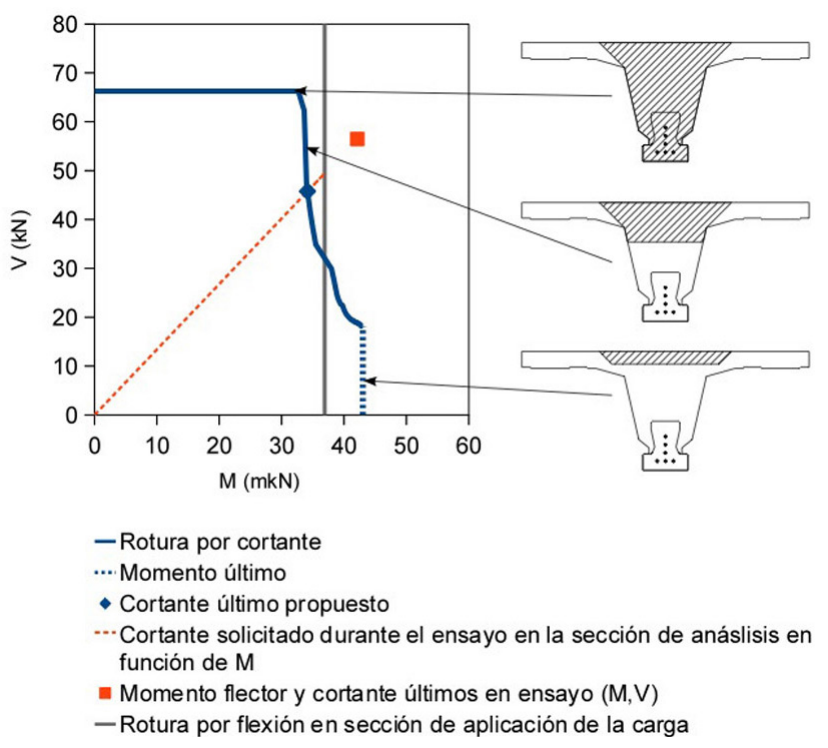

Figura 6. Interacción momento-cortante propuesta para la sección 29-T5-T12-CC4.

en la predicción del cortante último, ya que las predicciones de carga última a flexión concuerdan de forma adecuada con los resultados experimentales en el caso de los elementos que han roto a flexión. El motivo de ello puede estar en que la formulación a cortante de la Instrucción EHE-o8 es de origen empírico a partir de vigas ensayadas a cortante con otras características diferentes a las aquí estudiadas, y que no tiene en cuenta la interacción cortante-flexión ni el efecto beneficioso de las alas comprimidas.

Por otra parte, la Tabla 2 también presenta los cocientes entre las cargas de rotura previstas por la propuesta presentada en este artículo y la carga de rotura experimental, el promedio de estos cocientes es de 1,14 con una desviación estándar de 0,10 y un coeficiente de variación del 9,1\%. Estos valores son más adecuados que los de la Instrucción EHE-o8, y parten de un modelo mecánico racional fruto de la observación experimental, pero en el que no se incluye ningún ajuste a partir de la experimentación efectuada. Es necesario señalar que las estadísticas deben considerarse con precaución, especialmente las de la rotura a cortante, debido a que el número de ensayos es reducido.

En la Figura 7 se presentan tres gráficas en las que se compara la carga última predicha con la carga última experimental, para la Instrucción EHE-o8 en la primera gráfica, para el método de Zararis et al., en la segunda y para el modelo propuesto en la tercera. En esta figura se diferencian las roturas a cortante de las que se han producido a flexión. La Figura 7 muestra de forma clara el mejor comportamiento de la propuesta con respecto de la Instrucción EHE-08 y el modelo de Zararis et al.

\subsection{Comparación del modelo propuesto con los resultados experimentales en vigas con sección en $\mathbf{T}$}

En la Tabla 3 se muestra el resumen estadístico de resultados de los cocientes entre el cortante resistido en los ensayos de vigas con sección en $\mathrm{T}$ citadas en el apartado $4.2 \mathrm{y}$ el cortante predicho en la Instrucción EHE-o8, el predicho por el modelo Zararis et al. y el predicho por el modelo propuesto. 
Tabla 2. Resumen de resultados del modelo propuesto, para forjados de vigueta y bovedilla pretensada.

\begin{tabular}{|c|c|c|c|c|c|c|c|c|c|c|c|c|c|c|}
\hline \multirow[b]{2}{*}{ Serie } & \multirow[b]{2}{*}{ Viga } & \multicolumn{2}{|c|}{ Datos experimentales } & \multicolumn{5}{|c|}{ Cargas Últimas EHE-08 } & \multicolumn{6}{|c|}{ Cargas Últimas Propuesta } \\
\hline & & $\begin{array}{c}\text { Carga } \\
\text { Última } \\
\text { observada } \\
\text { kN }\end{array}$ & $\begin{array}{c}\text { Tipo de } \\
\text { rotura } \\
\text { observado }\end{array}$ & $\begin{array}{c}\text { a flexión } \\
\mathrm{kN}\end{array}$ & $\begin{array}{c}\text { a cortante } \\
\text { lado corto } \\
\text { kN }\end{array}$ & $\begin{array}{l}\text { más } \\
\text { desf. } \\
\mathrm{kN}\end{array}$ & $\begin{array}{l}\text { Tipo de } \\
\text { rotura } \\
\text { predicho }\end{array}$ & $\begin{array}{l}\text { Pexp/ } \\
\text { PEHE }\end{array}$ & $\begin{array}{c}\text { a flexión } \\
\mathrm{kN}\end{array}$ & $\begin{array}{l}\text { a cortante } \\
\text { lado corto } \\
\text { kN }\end{array}$ & $\begin{array}{c}\text { a cortante } \\
\text { lado largo } \\
\text { kN }\end{array}$ & $\begin{array}{l}\text { más } \\
\text { desf. } \\
\mathrm{kN}\end{array}$ & $\begin{array}{l}\text { Tipo de } \\
\text { rotura } \\
\text { predicho }\end{array}$ & $\begin{array}{l}\text { Pexp/ } \\
\text { Pprop }\end{array}$ \\
\hline A & $\begin{array}{c}\text { 24-T3-T12-SCC } \\
24-\mathrm{T}_{3}-\mathrm{T} 12-\mathrm{CC}_{4} \\
24-\mathrm{T} 3-\mathrm{T} 12-\mathrm{CC} 7\end{array}$ & $\begin{array}{c}64,78 \\
61,87 \\
70,00 \\
\end{array}$ & $\begin{array}{l}\mathrm{S} \\
\mathrm{S} \\
\mathrm{F}\end{array}$ & $\begin{array}{l}53,07 \\
65,91 \\
65,91 \\
\end{array}$ & $\begin{array}{l}45,61 \\
45,61 \\
45,61 \\
\end{array}$ & $\begin{array}{l}45,61 \\
45,61 \\
45,61 \\
\end{array}$ & $\begin{array}{l}\mathrm{S} \\
\mathrm{S} \\
\mathrm{S}\end{array}$ & $\begin{array}{l}1,42 \\
1,36 \\
1,53 \\
\end{array}$ & $\begin{array}{l}53,07 \\
65,91 \\
65,91 \\
\end{array}$ & $\begin{array}{l}49,88 \\
\mathbf{6 0 , 7 0} \\
\mathbf{6 1 , 0 2} \\
\end{array}$ & $\begin{array}{l}49,47 \\
62,23 \\
62,66 \\
\end{array}$ & $\begin{array}{l}49,47 \\
60,70 \\
61,02 \\
\end{array}$ & $\begin{array}{l}\text { SL } \\
\text { S } \\
\text { S }\end{array}$ & $\begin{array}{l}1,31 \\
1,02 \\
1,15 \\
\end{array}$ \\
\hline B & $\begin{array}{l}24-\mathrm{T}_{5}-\mathrm{T} 12-\mathrm{SCC} \\
24-\mathrm{T}_{5}-\mathrm{T} 12-\mathrm{CC} 4 \\
24-\mathrm{T} 5-\mathrm{T} 12-\mathrm{CC} 7\end{array}$ & $\begin{array}{c}81,85 \\
101,68 \\
107,55 \\
\end{array}$ & $\begin{array}{l}\text { SL } \\
\text { F } \\
\text { F }\end{array}$ & $\begin{array}{l}69,53 \\
95,58 \\
95,58 \\
\end{array}$ & $\begin{array}{l}53,67 \\
53,67 \\
53,67 \\
\end{array}$ & $\begin{array}{l}53,67 \\
53,67 \\
53,67 \\
\end{array}$ & $\begin{array}{l}S \\
S \\
S\end{array}$ & $\begin{array}{l}1,53 \\
1,89 \\
2,00 \\
\end{array}$ & $\begin{array}{l}\mathbf{6 9 , 5 3} \\
95,58 \\
95,58 \\
\end{array}$ & $\begin{array}{l}70,20 \\
\mathbf{8 5 , 3 2} \\
\mathbf{8 7 , 3 0} \\
\end{array}$ & $\begin{array}{l}70,55 \\
86,25 \\
87,92 \\
\end{array}$ & $\begin{array}{l}69,53 \\
85,32 \\
87,30 \\
\end{array}$ & $\begin{array}{l}\mathrm{F} \\
\mathrm{S} \\
\mathrm{S}\end{array}$ & $\begin{array}{l}1,18 \\
1,19 \\
1,23 \\
\end{array}$ \\
\hline $\mathrm{C}$ & $\begin{array}{l}29-\mathrm{T}_{3}-\mathrm{T}_{12}-\mathrm{SCC} \\
29-\mathrm{T}_{3}-\mathrm{T}_{12}-\mathrm{CC}_{4} \\
29-\mathrm{T}_{3}-\mathrm{T}_{12}-\mathrm{CC}_{7}\end{array}$ & $\begin{array}{l}72,00 \\
72,19 \\
71,87 \\
\end{array}$ & $\begin{array}{l}\mathrm{F} \\
\mathrm{F} \\
\mathrm{F}\end{array}$ & $\begin{array}{l}63,80 \\
72,66 \\
72,66 \\
\end{array}$ & $\begin{array}{l}54,71 \\
54,71 \\
54,71 \\
\end{array}$ & $\begin{array}{l}54,71 \\
54,71 \\
54,71 \\
\end{array}$ & $\begin{array}{l}\mathrm{S} \\
\mathrm{S} \\
\mathrm{S}\end{array}$ & $\begin{array}{l}1,32 \\
1,32 \\
1,31 \\
\end{array}$ & $\begin{array}{l}63,80 \\
72,66 \\
72,66 \\
\end{array}$ & $\begin{array}{l}55,72 \\
65,06 \\
65,87 \\
\end{array}$ & $\begin{array}{l}56,31 \\
68,70 \\
70,00 \\
\end{array}$ & $\begin{array}{l}55,72 \\
65,06 \\
65,87 \\
\end{array}$ & $\begin{array}{l}\mathrm{S} \\
\mathrm{S} \\
\mathrm{S}\end{array}$ & $\begin{array}{l}1,29 \\
1,11 \\
1,09 \\
\end{array}$ \\
\hline $\mathrm{D}$ & $\begin{array}{l}29-\mathrm{T}_{5}-\mathrm{T} 12-\mathrm{SCC} \\
29-\mathrm{T} 5-\mathrm{T} 12-\mathrm{CC}_{4} \\
29-\mathrm{T} 5-\mathrm{T} 12-\mathrm{CC}_{7}\end{array}$ & $\begin{array}{c}96,18 \\
90,32 \\
107,05 \\
\end{array}$ & $\begin{array}{l}\text { SL } \\
\text { S } \\
\text { F }\end{array}$ & $\begin{array}{r}86,25 \\
104,48 \\
104,48 \\
\end{array}$ & $\begin{array}{l}64,81 \\
64,81 \\
64,81 \\
\end{array}$ & $\begin{array}{l}64,81 \\
64,81 \\
64,81 \\
\end{array}$ & $\begin{array}{l}\mathrm{S} \\
\mathrm{S} \\
\mathrm{S}\end{array}$ & $\begin{array}{l}1,48 \\
1,39 \\
1,65 \\
\end{array}$ & $\begin{array}{c}86,25 \\
104,48 \\
104,48 \\
\end{array}$ & $\begin{array}{l}\mathbf{7 6 , 9 5} \\
92,83 \\
\mathbf{9 4 , 1 7} \\
\end{array}$ & $\begin{array}{c}78,52 \\
\mathbf{9 2 , 6 2} \\
94,74 \\
\end{array}$ & $\begin{array}{l}76,95 \\
92,62 \\
94,17 \\
\end{array}$ & $\begin{array}{c}\text { S } \\
\text { SL } \\
\text { S }\end{array}$ & $\begin{array}{l}1,25 \\
0,98 \\
1,14 \\
\end{array}$ \\
\hline $\mathrm{E}$ & $\begin{array}{l}24-\mathrm{T}_{3}-\mathrm{T}_{20}-\mathrm{SCC} \\
24-\mathrm{T}_{3}-\mathrm{T}_{20}-\mathrm{CC}_{4} \\
24-\mathrm{T}_{3}-\mathrm{T}_{20}-\mathrm{CC}_{7}\end{array}$ & $\begin{array}{l}56,68 \\
61,60 \\
60,90 \\
\end{array}$ & $\begin{array}{l}\mathrm{F} \\
\mathrm{F} \\
\mathrm{F}\end{array}$ & $\begin{array}{l}52,91 \\
59,50 \\
59,50 \\
\end{array}$ & $\begin{array}{l}30,69 \\
30,69 \\
30,69 \\
\end{array}$ & $\begin{array}{l}30,69 \\
30,69 \\
30,69 \\
\end{array}$ & $\begin{array}{l}\mathrm{S} \\
\mathrm{S} \\
\mathrm{S}\end{array}$ & $\begin{array}{l}1,85 \\
2,01 \\
1,98 \\
\end{array}$ & $\begin{array}{l}52,91 \\
59,50 \\
59,50 \\
\end{array}$ & $\begin{array}{l}47,29 \\
\mathbf{5 4 , 4 2} \\
\mathbf{5 4 , 7 5} \\
\end{array}$ & $\begin{array}{l}\mathbf{4 7 , 1 4} \\
56,26 \\
56,58 \\
\end{array}$ & $\begin{array}{l}47,14 \\
54,42 \\
54,75 \\
\end{array}$ & $\begin{array}{c}\text { SL } \\
\text { S } \\
\text { S }\end{array}$ & $\begin{array}{l}1,20 \\
1,13 \\
1,11 \\
\end{array}$ \\
\hline $\mathrm{F}$ & $\begin{array}{l}24-\mathrm{T}_{5}-\mathrm{T}_{20}-\mathrm{SCC} \\
24-\mathrm{T}_{5}-\mathrm{T}_{20}-\mathrm{CC}_{4} \\
24-\mathrm{T}_{5}-\mathrm{T}_{20}-\mathrm{CC}_{7}\end{array}$ & $\begin{array}{l}76,50 \\
90,00 \\
92,74 \\
\end{array}$ & $\begin{array}{l}\text { SL } \\
\mathrm{F} \\
\mathrm{F}\end{array}$ & $\begin{array}{l}74,01 \\
87,55 \\
87,55 \\
\end{array}$ & $\begin{array}{l}35,15 \\
35,15 \\
35,15 \\
\end{array}$ & $\begin{array}{l}35,15 \\
35,15 \\
35,15 \\
\end{array}$ & $\begin{array}{l}\mathrm{S} \\
\mathrm{S} \\
\mathrm{S}\end{array}$ & $\begin{array}{l}2,18 \\
2,56 \\
2,64 \\
\end{array}$ & $\begin{array}{l}74,01 \\
87,55 \\
87,55 \\
\end{array}$ & $\begin{array}{l}65,01 \\
78,32 \\
79,55 \\
\end{array}$ & $\begin{array}{l}68,04 \\
79,74 \\
81,54 \\
\end{array}$ & $\begin{array}{l}65,01 \\
78,32 \\
79,55 \\
\end{array}$ & $\begin{array}{l}\mathrm{S} \\
\mathrm{S} \\
\mathrm{S} \\
\end{array}$ & $\begin{array}{l}1,18 \\
1,15 \\
1,17 \\
\end{array}$ \\
\hline G & $\begin{array}{l}29-\mathrm{T}_{3}-\mathrm{T}_{20}-\mathrm{SCC} \\
29-\mathrm{T}_{3}-\mathrm{T}_{20}-\mathrm{CC}_{4} \\
29-\mathrm{T}_{3}-\mathrm{T}_{20}-\mathrm{CC}_{7}\end{array}$ & $\begin{array}{l}67,82 \\
65,30 \\
66,98 \\
\end{array}$ & $\begin{array}{l}\mathrm{F} \\
\mathrm{F} \\
\mathrm{F}\end{array}$ & $\begin{array}{l}61,33 \\
67,51 \\
67,51 \\
\end{array}$ & $\begin{array}{l}36,20 \\
36,20 \\
36,20\end{array}$ & $\begin{array}{l}36,20 \\
36,20 \\
36,20 \\
\end{array}$ & $\begin{array}{l}\mathrm{S} \\
\mathrm{S} \\
\mathrm{S}\end{array}$ & $\begin{array}{l}1,87 \\
1,80 \\
1,85 \\
\end{array}$ & $\begin{array}{l}61,33 \\
67,51 \\
67,51 \\
\end{array}$ & $\begin{array}{l}53,89 \\
\mathbf{6 0 , 2 2} \\
\mathbf{6 0 , 7 2} \\
\end{array}$ & $\begin{array}{c}\mathbf{5 3 , 8 8} \\
64,72 \\
65,50 \\
\end{array}$ & $\begin{array}{l}53,88 \\
60,22 \\
60,72 \\
\end{array}$ & $\begin{array}{c}\text { SL } \\
\text { S } \\
\text { S } \\
\end{array}$ & $\begin{array}{l}1,26 \\
1,08 \\
1,10 \\
\end{array}$ \\
\hline $\mathrm{H}$ & $\begin{array}{l}29-\mathrm{T}_{5}-\mathrm{T}_{20}-\mathrm{SCC} \\
29-\mathrm{T}_{5}-\mathrm{T}_{20}-\mathrm{CC}_{4} \\
29-\mathrm{T}_{5}-\mathrm{T}_{20}-\mathrm{CC}_{7}\end{array}$ & $\begin{array}{l}96,30 \\
93,00 \\
94,00\end{array}$ & $\begin{array}{l}\mathrm{F} \\
\mathrm{F} \\
\mathrm{F}\end{array}$ & $\begin{array}{l}85,76 \\
97,51 \\
97,51 \\
\end{array}$ & $\begin{array}{l}41,78 \\
41,78 \\
41,78 \\
\end{array}$ & $\begin{array}{l}41,78 \\
41,78 \\
41,78 \\
\end{array}$ & $\begin{array}{l}S \\
S \\
S\end{array}$ & $\begin{array}{l}2,30 \\
2,23 \\
2,25 \\
\end{array}$ & $\begin{array}{l}85,76 \\
97,51 \\
97,51 \\
\end{array}$ & $\begin{array}{l}75,92 \\
86,66 \\
87,20 \\
\end{array}$ & $\begin{array}{l}77,16 \\
88,07 \\
89,84 \\
\end{array}$ & $\begin{array}{l}75,92 \\
86,66 \\
87,20 \\
\end{array}$ & $\begin{array}{l}\mathrm{S} \\
\mathrm{S} \\
\mathrm{S}\end{array}$ & $\begin{array}{l}1,27 \\
1,07 \\
1,08 \\
\end{array}$ \\
\hline 2007 & $\begin{array}{l}30-\mathrm{T}_{5}-\mathrm{T} 12-\mathrm{CC}_{5} \\
25-\mathrm{T} 5-\mathrm{T} 12-\mathrm{CC}_{5} \\
20-\mathrm{T} 5-\mathrm{T} 12-\mathrm{CC} 8\end{array}$ & $\begin{array}{l}66,53 \\
56,21 \\
72,31 \\
\end{array}$ & $\begin{array}{l}S \\
S \\
S\end{array}$ & $\begin{array}{l}76,90 \\
71,94 \\
66,33 \\
\end{array}$ & $\begin{array}{l}56,54 \\
46,14 \\
36,37 \\
\end{array}$ & $\begin{array}{l}56,54 \\
46,14 \\
36,37 \\
\end{array}$ & $\begin{array}{l}\mathrm{S} \\
\mathrm{S} \\
\mathrm{S} \\
\end{array}$ & $\begin{array}{l}1,18 \\
1,22 \\
1,99 \\
\end{array}$ & $\begin{array}{l}76,90 \\
71,94 \\
66,33 \\
\end{array}$ & $\begin{array}{l}68,61 \\
64,88 \\
61,85 \\
\end{array}$ & $\begin{array}{l}73,27 \\
67,29 \\
63,56 \\
\end{array}$ & $\begin{array}{l}68,61 \\
64,88 \\
61,85 \\
\end{array}$ & $\begin{array}{l}\mathrm{S} \\
\mathrm{S} \\
\mathrm{S} \\
\end{array}$ & $\begin{array}{c}0,97 \\
0,87 \\
1,17 \\
\end{array}$ \\
\hline & & & & & & & Promedio $=$ & 1,78 & & & & & Promedio $=$ & 1,14 \\
\hline & & & & & & & Desv. Est.= & 0,41 & & & & & Desv. Est.= & 0,10 \\
\hline & & & & & & & C.0.V = & 23,2 & & & & & C. $0 . \mathrm{V}=$ & 9,1 \\
\hline
\end{tabular}

Si bien en la Tabla 3 se puede observar como el promedio de los cocientes $V_{\text {exp }} / V_{\text {pred }}$ para el modelo propuesto está más del lado de la seguridad que el de la Instrucción EHE-o8 y el modelo de Zararis et al., el coeficiente de variación es menor en el modelo propuesto que en los otros dos modelos estudiados. Además, el resto de los valores mostrados para el modelo propuesto en la Tabla 3 se presentan en un rango conveniente para un modelo de diseño. Por todo ello, el modelo propuesto se muestra como un modelo adecuado para el diseño de vigas de hormigón armado con sección en T. El hecho de que la extrapolación del modelo a otras vigas, similares pero no iguales, prediga de forma satisfactoria las resistencias últimas a cortante, demuestra la adecuación del modelo mecánico sobre el que se asienta. Cabe destacar que en ningún caso, en el desarrollo de esta investigación, se ha tratado de optimizar el ajuste para estas vigas en $\mathrm{T}$, sino que se trata sencillamente de una extrapolación, ya que el foco del trabajo de investigación han sido los forjados de vigueta y bovedilla.

En la Figura 8 se compara el cortante experimental resistido en los ensayos de las 188 vigas con sección en T con el cortante predicho, en la figura se muestran en líneas entrecortadas las desviaciones del $15 \%$. En la figura se muestra que el modelo propuesto es un modelo adecuado para el dimensionamiento a cortante de las vigas con sección en $\mathrm{T}$, mostrando una precisión similar al del modelo de Zararis et al. y mayor que el la Instrucción EHE-o8, si bien se presenta más por el lado de la seguridad. Se observa que la Instrucción EHE-o8 tiene una menor precisión en este tipo de vigas en T. Esto es probablemente debido a que la Instrucción EHE-o8 no considera ningún incremento de resistencia por efecto de las alas comprimidas en vigas en sección en $\mathrm{T}$.

En la Figura 9 se muestra la relación entre la cuantía del armado longitudinal $\rho_{l}$ y los cocientes $\mathrm{V}_{\text {exp }} / \mathrm{V}_{\text {pred }}$ de los ensayos de las vigas con sección en $\mathrm{T}$ para el modelo de Zararis et al. $\mathrm{y}$ el modelo propuesto. En ella se puede observar como el modelo propuesto se acerca a la unidad para cuantías bajas y se aleja, por el lado de la seguridad, para cuantías altas. Este fenómeno tiene sentido debido a que el modelo está basado en que el mecanismo principal de transmisión del cortante es el de las tensiones tangenciales transmitidas a través de la zona comprimida. Al aumentar la cuantía, la transmisión de tensiones a través de la fisura y mediante el efecto pasador aumenta, por lo que la seguridad del modelo propuesto aumenta al no tener en cuenta esos mecanismos resistentes. En el modelo de Zararis et al. no se observa la misma tendencia, probablemente debido a la simplificación de la posición de la fibra neutra propuesta por los autores.

El modelo propuesto se presenta especialmente adecuado para vigas con sección en $\mathrm{T}$ con cuantías $\rho_{l}$ bajas, y por lo tanto para las secciones resistentes de los forjados de vigueta pretensada y bovedilla que tienen las citadas características.

\section{CONCLUSIONES}

En el presente estudio se han modificado algunas de las simplificaciones planteadas en el modelo de Zararis et al. para la 
EHE-08 $\quad R^{2}=0,21$

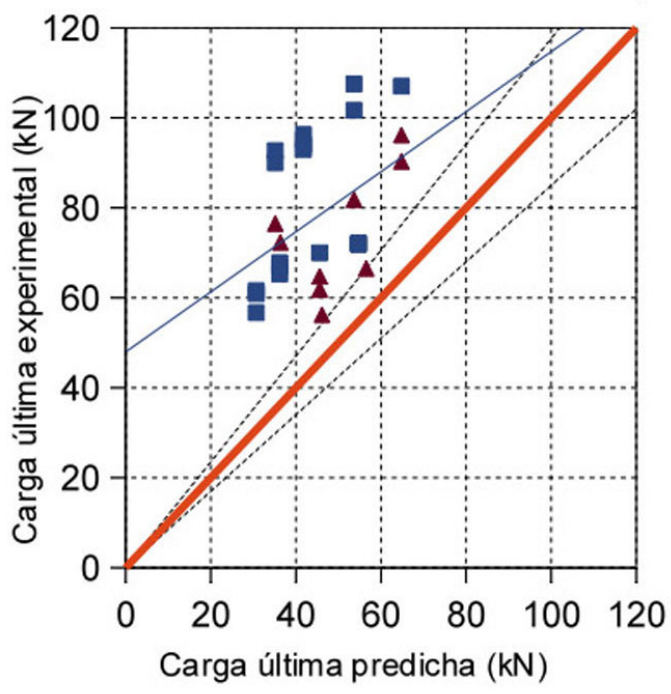

Propuesta $\quad R^{2}=0,81$

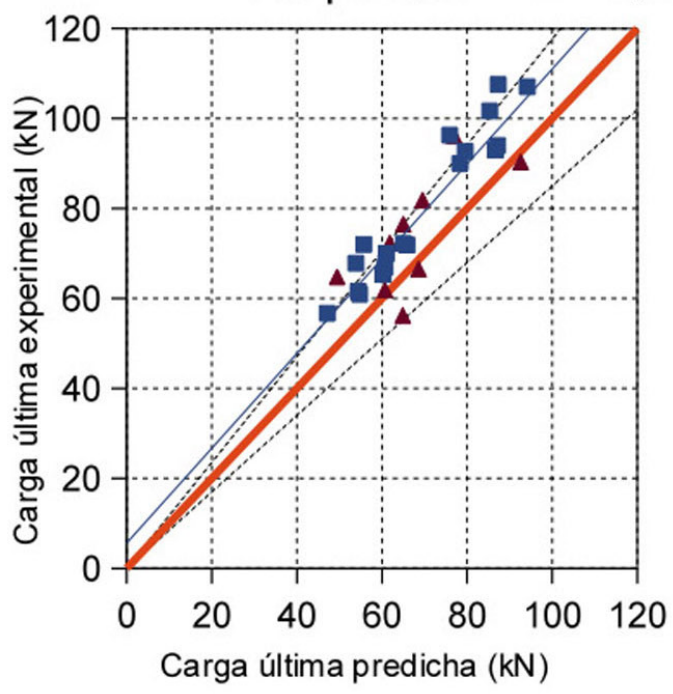

Zararis

$\mathrm{R}^{2}=0,75$

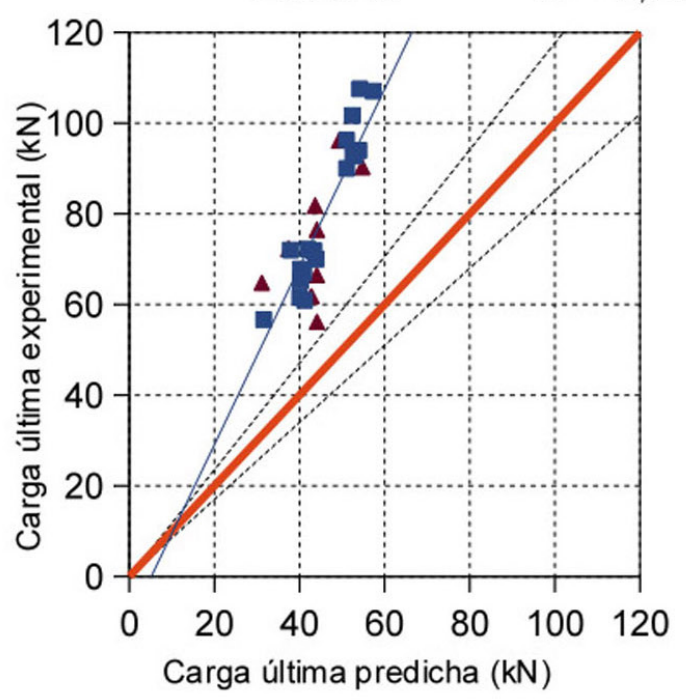

- Roturas a flexión $\Delta$ Roturas a cortante

- Línea de tendencia -....15\%

Figura 7. Comparativa entre ensayos experimentales y predicciones de la EHE-08 y el modelo racional propuesto.
EHE-08

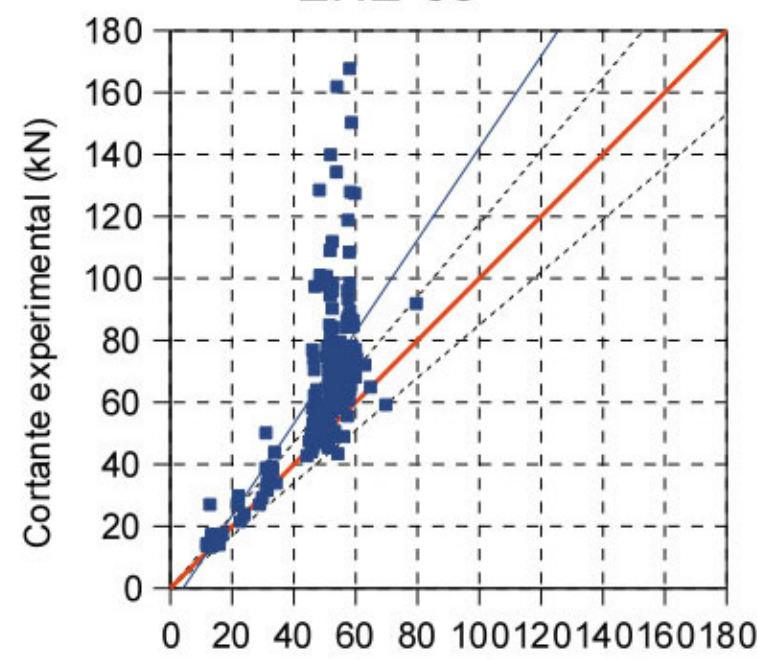

$\mathrm{R}^{2}=0,55 \quad$ Cortante predicho $(\mathrm{kN})$

\section{Zararis}

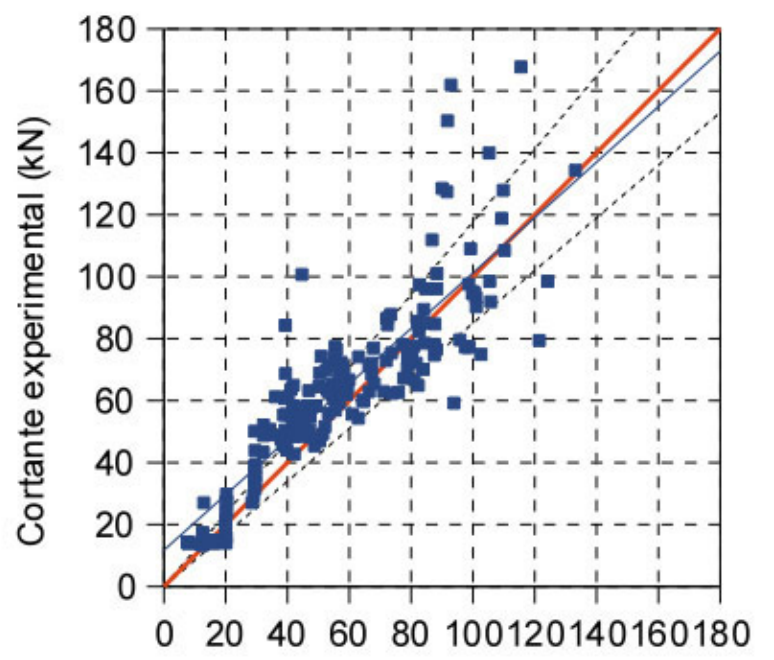

$\mathrm{R}^{2}=0,77 \quad$ Cortante predicho $(\mathrm{kN})$

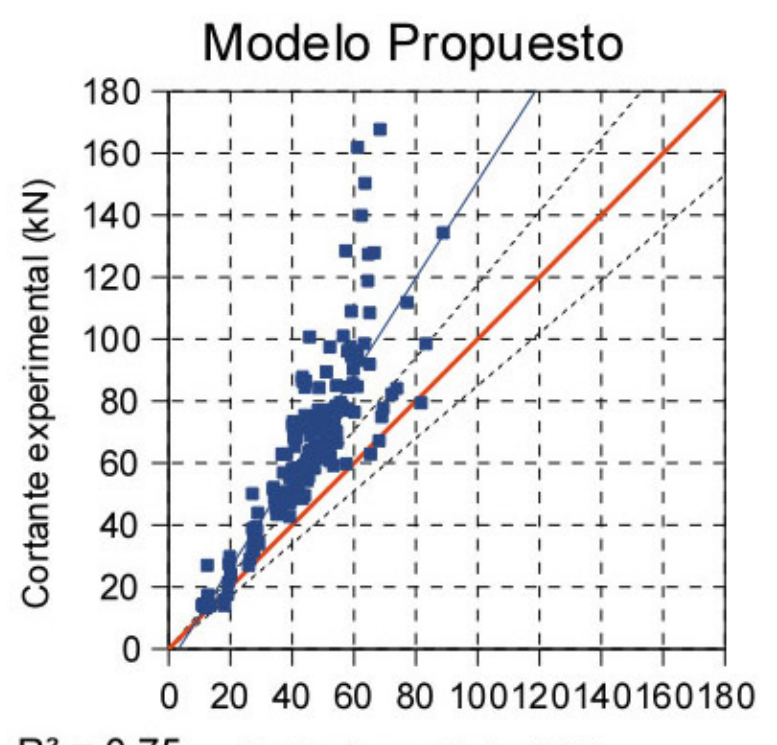

$\mathrm{R}^{2}=0,75$ Cortante predicho $(\mathrm{kN})$

Figura 8. Comparativa entre ensayos experimentales y predicciones de la Instrucción EHE-o8, el modelo Zararis y el modelo propuesto. 
Tabla 3. Resumen estadístico de resultados de la Instrucción EHE-o8, del modelo de Zararis et al. y del modelo propuesto, para vigas con sección en T extraídas de la bibliografía.

\begin{tabular}{|l|c|c|c|}
\hline Normativa/Modelo & EHE-o8 & Zararis & Propuesta \\
\hline No $^{\circ}$ de vigas & 188 & 188 & 188 \\
\hline Promedio Vexp/Vpred & 1,32 & 1,16 & 1,41 \\
\hline Mediana & 1,21 & 1,14 & 1,36 \\
\hline Desv. Est. & 0,39 & 0,27 & 0,29 \\
\hline Coef. Var. (\%) & 29,33 & 23,55 & 20,65 \\
\hline Mínimo & 0,80 & 0,63 & 0,77 \\
\hline Máximo & 3,00 & 2,26 & 2,65 \\
\hline Percentil 5\% & 0,94 & 0,80 & 1,09 \\
\hline Percentil 95\% & 2,11 & 1,70 & 1,96 \\
\hline
\end{tabular}

evaluación de la resistencia a cortante de elementos de hormigón armado, con el fin de poder evaluar la resistencia a cortante de forjados de vigueta pretensada y bovedilla, y vigas con sección en T. Las conclusiones extraídas de la aplicación del modelo propuesto para el cálculo a estado límite último de cortante son:

- Se ha presentado un modelo mecánico basado en que el principal mecanismo resistente a cortante son las tensiones tangenciales en la zona comprimida a flexión. El modelo se ha mostrado como el más adecuado de los comparados para el dimensionamiento de forjados de vigueta pretensada y bovedilla, y su extrapolación para vigas con sección en T se muestra como un método de diseño correcto.

- La Instrucción EHE-o8 proporciona valores muy del lado de la seguridad en las roturas a cortante para forjados de vigueta pretensada y bovedilla.

- El modelo presentado predice de forma más adecuada la resistencia a cortante de los forjados de vigueta y bovedilla ensayados que la Instrucción EHE-o8.

- El modelo mecánico presentado es de base racional y es capaz de responder al efecto que produce la variación del espesor de la capa de compresión detectado en las campañas experimentales sobre forjados de vigueta pretensada y bovedilla.

- El modelo presentado predice mejor la resistencia a cortante de las vigas con sección en T con baja cuantía que el de Zararis et al., por lo que se muestra muy adecuado para los forjados de vigueta pretensada y bovedilla.

\section{AGRADECIMIENTOS}

Este artículo se ha desarrollado en el ámbito del proyecto de investigación «Comportamiento en servicio y capacidad resistente de piezas compuestas por varios hormigones construidas evolutivamente. Aplicación a forjados y vigas de edificación» (BIA2007-60197) financiado por el Ministerio de
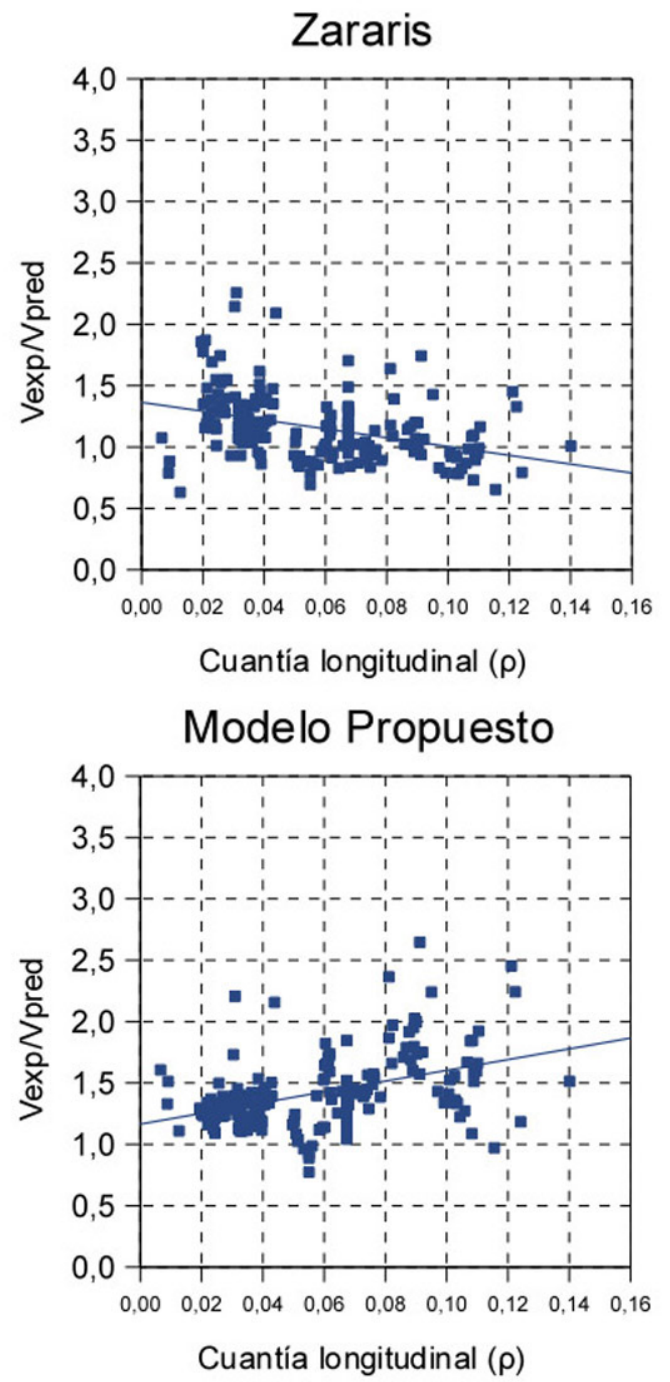

- Ensayos vigas sección en T

Regresión lineal para Ensayos vigas sección en T

Figura 9. Relación entre la cuantía longitudinal y los cocientes $\mathrm{V}_{\text {exp }}$ / $\mathrm{V}_{\text {pred }}$, en el modelo Zararis y el modelo propuesto.

Educación y Ciencia del Gobierno de España y del proyecto «Materiales inteligentes en hormigón estructural. Aplicación de aleaciones con memoria de forma como refuerzo a cortante de elementos lineales» (BIA2012-31432) cofinanciado por el Ministerio de Economía y Competitividad del Gobierno de España y por el Fondo Europeo de Desarrollo Regional (FEDER). Agradecer a la empresa constructora y de prefabricados PASTOR SA la fabricación de los especímenes y que haya facilitado su laboratorio para la realización de los ensayos.

\section{REFERENCIAS}

(1) Ribas, C. (2013). Resistencia a cortante de los forjados de vigueta pretensada y bovedilla (Tesis doctoral). Barcelona: UPC-Departamento de Ingeniería de la Construcción.

(2) Ribas, C., Cladera, A. (2013). Experimental study on shear strength of beam-and-block floors. Engineering Structures. Elsevier, 57: 428-442. doi: http://dx.doi.org/10.1016/j.engstruct.2013.10.001.

(3) Comisión Permanente del Hormigón. (2008). EHE-o8. Instrucción de Hormigón Estructural.

(4) Al-Alusi, A. (1957). Diagonal tension strength of reinforced concrete T-beams with varying shear span. ACI Journal Proceedings, 53: 1067-1077.

(5) Swamy, R., Qureshi, S. (1974). An ultimate shear strength theory for reinforced concrete T-beams without web reinforcement. Materials and Structures, 7: 181-189. 
(6) Zararis, I. P., Karaveziroglou, M. K., Zararis, P. D. (2006). Shear strength of reinforced concrete T-beams. ACI structural journal, 103: 693-700.

(7) Zararis, P. D., Papadakis, G. C. (2001). Diagonal shear failure and size effect in RC beams without web reinforcement. Journal of structural engineering, 127(7): 733-742, doi: http://dx.doi.org/10.1061/(ASCE)0733-9445(2001)127:7(733).

(8) Kotsovos, M. D., Bobrowski, J., Eibl, J. (1987). Behaviour of reinforced concreteT-beams in shear. The Structural Enginner, 65B: 1-10.

(9) Parra, C., Martínez-Conesa, E., Valcuende, M., Garrido, A. (2012). Análisis de métodos para evaluar el refuerzo a esfuerzo cortante con CFRP en vigas de hormigón armado. Informes de la Construcción, 64(526): 197-206, doi: http://dx.doi. org/10.3989/ic.11.007.

(10) Marí, A., Cladera, A., Oller, E., Bairán, J. (2014). Shear design of FRP reinforced concrete beams without transverse reinforcement. Composites: Part B. Elsevier, 57: 228-241, doi: http://dx.doi.org/10.1016/j.compositesb.2013.10.005.

(11) Bairán, J., Mari, A., Mohr, S. (2010). Behavior of reinforced concrete under normal and tangential forces by means of Total-Interaction-Nonlinear-Sectional-Analysis (TINSA). Informes de la Construcción, 62(518): 65-77, doi: http:// dx.doi.org/10.3989/ic.09.021.

(12) Fenwick, R., Paulay, T. (1968). Mechanisms of shear resistance of concrete beams. Journal of Structural Division, 94: 2325-2350.

(13) Walraven, J. (1981). Fundamental analysis of agregate interlock. Journal of Structural Division - Proceedings of the ASCE, 107(11): 2245-2270.

(14) Ferguson, P. M., Thompson, J. N. (1953). Diagonal Tension in T-Beams Without Stirrups. ACI Journal Proceedings, 49, 665-675.

(15) Placas, A., Regan, P. E. (1971). Shear Failure of Reinforced Concrete Beams. Journal Proceedings. 68: $763-773$.

(16) Kani, M., Huggins, M., Wittkopp, R. (1979). Kani on Shear in Reinforced Concrete. Toronto: University of Toronto Dept. of Civil Engineering.

(17) Taylor, M. A., El-Hammasi, S. (1980). Web Cracking Behavior of Beams Using Welded Wire Fabric as Shear Reinforcement. ACI Journal, Proceedings, 77(1): 12-17, doi: http://dx.doi.org/ 10.14359/6985.

(18) Palaskas, M. N., Attiogbe, E. K., Darwin, D. (1981). Shear of Lightly Reinforced T-Beams. ACI Journal Proceedings, 78(6): 447-455, doi: http://dx.doi.org/ 10.14359/10528.

(19) Collins, M. P., Bentz, E. C., Sherwood, E. G. (2008). Where is shear reinforcement required? Review of research results and design procedures. ACI Structural Journal, 105(5): 590-600, doi: http://dx.doi.org/ 10.14359/19942.

(20) Collins, M. P., Mitchell, D. (1991). Prestressed concrete structures. USA: Prentice Hall. 